\title{
Estima corporal en gimnastas adolescentes de dos disciplinas coreográficas: gimnasia rítmica y gimnasia acrobática
}

\author{
Mercedes VERNETTA, Isabel MONTOSA, y Eva PELÁEZ
}

Departamento Educación Física y Deportiva. Universidad de Granada

(Recibido, 5 de Junio de 2018; Aceptado, 11 de Julio de 2018)

RESUMEN: El objetivo del estudio fue analizar y comparar la estima corporal (EC) entre gimnastas de rítmica (GR) y gimnastas de acrobática (GA) y su relación con el IMC y el perímetro de cintura. La EC fue medida con el Body Esteem Scale for Children (BES-C). El IMC y el perímetro de cintura de todas gimnastas fue de $17.47 \mathrm{~kg} / \mathrm{m} 2( \pm 2.36)$ y $61.13 \mathrm{~cm}( \pm 4.36)$ respectivamente, estando todas en delgadez aceptable según los valores de referencia y un perímetro de cintura bajo, existiendo diferencias significativas entre ambas disciplinas $(\mathrm{p}<.05)$. Los resultados del BES-C muestran buena EC con una puntuación media de $10.64 \pm 1.0$ en GA y 9.48 \pm 1.95 en GR. El 100\% de las gimnastas de acrobática respondieron positivamente a los ítem "Me siento satisfecho con mi físico", "Me gusta como salgo en las fotos", "Me gusto en el espejo" y "Estoy orgullosa de mi cuerpo". Mientras que las de rítmica obtuvieron porcentajes ligeramente más bajos $(84.4 \%, 72.7 \%, 97.1 \%$ y 93.9\%, respectivamente). No existieron diferencias significativas en ninguna pregunta a excepción de " Me gusta como salgo en las fotos" y "Me gustaría tener un físico mejor" $(\mathrm{p}<.05)$ donde 9 de las 33 gimnastas de rítmica no le gusta como sale en las fotos y 14 de las 34 de acrobática le gustaría tener un físico mejor. Se encontraron relaciones significativas de signo positivo, entre IMC-Perímetro de cintura, IMC-Edad y Perímetro de cintura-Edad $(\mathrm{p}<.001)$. Igualmente, existió relación entre la estima corporal-IMC $(\mathrm{p}<.05)$. Las gimnastas de ambas disciplinas presentan buena EC con diferencias significativas $(\mathrm{p}<.05)$. Todas en general tienen buena opinión sobre su aspecto y su físico. La estima corporal de las de acrobática se asoció con un IMC saludable ( $<<.05)$.

Palabras clave: gimnasia rítmica, gimnasia acrobática, estima corporal, test BES-C.

\section{Body-esteem in adolescent gymnasts of two choreographic disciplines: rhythmic gymnastics and acrobatic gymnastics}

ABSTRACT: The objective of the study was to analyze and compare body esteem (BE) between
rhythmic gymnasts (RG) and acrobatic gymnasts (AG) and their relationship with BMI and waist
circumference. The BE was measured with the Body Esteem Scale for Children (BES-C). The BMI and
the waist circumference of all gymnasts was $17.47 \mathrm{~kg} / \mathrm{m} 2( \pm 2.36)$ and $61.13 \mathrm{~cm}( \pm 4.36)$ respectively,
all being in acceptable thinness according to the reference values and a low waist circumference, there
being significant differences between both disciplines $(\mathrm{p}<.05)$. The results of the BES-C show good
EC with an average score of $10.64 \pm 1.0$ in GA and $9.48 \pm 1.95$ in GR. 100\% of the acrobatic gymnasts
responded positively to the items "I feel satisfied with my physique", "I like how I look in the photos",
"I like myself in the mirror" and "I am proud of my body". While the rhythmic ones obtained slightly 
lower percentages $(84.4 \%, 72.7 \%, 97.1 \%$ and $93.9 \%$, respectively). There were no significant differences in any questions except for "I like how I look in the photos" and "I would like to have a better physique" $(\mathrm{p}<.05)$ where 9 of the 33 rhythmic gymnasts do not like how it appears in the photos and 14 of the 34 of aerobics would like to have a better physique. Significant positive relationships were found, between BMI-waist circumference, BMI-Age and waist circumference-Age $(\mathrm{p}<.001)$. body estimation-BMI $(\mathrm{p}<.05)$.The gymnasts of both disciplines show good EC with significant differences between the two $(\mathrm{p}<.05)$. All in general have a good opinion about their appearance and their physique. Acrobatic was associated with a healthy BMI $(p<.05)$.

Keywords: Rhythmic gymnastics, acrobatic gymnastics, body esteem, test BES-C.

Correspondencia: Isabel Montosa Mirón, Departamento Educación Física y Deportiva, Universidad de Granada, Dirección C/ El Tejar, 6. C.P. 18329 El Jau- Granada. E-mail: isabelmontosa@gmail.com

\section{Introducción}

La gimnasia rítmica (GR) y la gimnasia acrobática (GA) son deportes con gran componente estético, de ahí que la imagen corporal sea un factor clave en los mismos ( Aguilera, 2016; Taboada-iglesias, Vernetta \& Gutiérrez-Sánchez, 2017; Vernetta, Fernández, López-Bedoya, Gómez-Landero \& Oña, 2011).

La GR es un deporte que implica importantes demandas físicas y el aprendizaje de elementos técnicos complejos, siendo el desarrollo de diversas capacidades físicas tales como: la flexibilidad, la fuerza explosiva, la coordinación, equilibrio y agilidad...etc., fundamentales para que la gimnasta logre un alto nivel de rendimiento, (Bobo-Arce \& Méndez-Rial, 2013; Douda, Toubekis, Avloniti y Tokmakidis, 2008; Vernetta, Montosa, Beas-Jiménez y López-Bedoya, 2017).

En cuanto a la GA es la última disciplina gimnástica integrada en la Federación Internacional de Gimnasia en 1999. Se trata de un deporte sociomotriz por excelencia de naturaleza coreográfica, donde se compiten en parejas o grupos sin utilización de ningún material, mediante la realización de movimientos individuales y cooperativos (Vernetta, López \& Gutierrez 2008).

Por otro lado, ambos deportes exigen una iniciación y especialización temprana con gran volumen de entrenamiento, siendo la adolescencia una de las etapas claves a nivel competitivo. Teniendo en cuenta que es en esta etapa cuando se produce innumerables cambios físicos y emocionales, es crucial prestarle mucha atención, ya que los problemas relacionados con la estima corporal pueden aumentar, debido a una preocupación excesiva por el aspecto físico y un peso bajo, para obtener éxito deportivo en la dimensión artística (Salazar, 2008; SaucedoMolina, Rodríguez, Oliva, Villarreal, León \& Fernández 2015).

Esta preocupación por una imagen corporal esbelta y ligera, propio de estos deportes estéticos, puede acarrear posibles desórdenes de alimentación o trastornos de conductas alimentarias (Nordin, Harris \& Cumming, 2003).

Tras revisar los estudios existentes sobre la EC, la mayoría de estos trabajos se han desarrollado en gimnastas de artística y rítmica (Camacho, Fernández \& Rodríguez, 2006; Campos, Pérez-Fabello \& Díaz, 2000; Esnaola, 2005; Hernández-Alcántara, AréchigaViramontes \& Prado, 2009; Vernetta el al., 2011), existiendo pocos en gimnasia acrobática Peláez \& Vernetta (2018), y ninguno que comparen la GR y GA. Aunque es cierto que ambas disciplinas gimnásticas comparten algunos puntos en común: dimensión coreográfica, soporte musical, gran dificultad técnica...etc., sin embargo, cada una de ellas tienen ciertas 
peculiaridades que las identifican, de ahí la importancia de realizar este trabajo. Estas diferencias están marcadas entre otras por el tipo de ejercicio y elementos en competición, el tiempo de los ejercicios, el número de integrantes siendo éste último quizás su mayor diferenciación, ya que la GA es un deporte sociomotriz por excelencia. Se necesita siempre la presencia de dos a cuatro competidores que sincronizan sus acciones motrices siempre en contacto corporal para conseguir la realización de figuras o pirámides humanas (Vernetta et al., 2008). Mientras que en GR las competidoras participan de forma individual, y si lo hacen en conjunto, generalmente, sus acciones de comunicación directa, están basadas en la manipulación de aparatos e intercambio de los mismos sin contacto corporal entre unas y otras.

Por otro lado, existen características antropométricas determinantes en las gimnastas de ambos deporte, como el peso y porcentaje de grasa bajo (Klentrou \& Plyley 2003.; ÁvilaCarvalho, Klentrou, Palomero \& Lebre 2012; Taboada-Iglesias et al., 2017).

No obstante las gimnastas de rítmica se caracterizan por un somatotipo con predominio del componente ectomórfico según Purenovic-Ivanovic \& Popovic ( 2014), mientras que en las de acrobática predomina el componente mesomórfico-ectomórfico (Taboada et al., 2017).

Además teniendo en cuenta que son deportes, donde la presión del entrenador y el índice de masa corporal (IMC), junto con el umbral competitivo, pueden ocasionar mucha preocupación por el peso, ocasionando posibles alteraciones de su imagen corporal, surge la necesidad de su estudio (Hernández-Alcántara et al., 2009).

Por ello, el objetivo fue analizar y comparar la estima corporal entre gimnastas de ambas disciplinas y su relación con el IMC y perímetro de cintura.

\section{Método}

\section{Diseño y participantes}

Estudio descriptivo, transversal y comparativo, a 67 gimnastas ( 34 de acrobática y 33 de rítmica); edad $12.36( \pm 2.34)$, peso $40.08 \mathrm{Kg}( \pm 10.13)$ y altura $1.50 \mathrm{~m}( \pm 0.11)$ de ámbito nacional. Las participantes presentaban una experiencia media de entrenamiento superior a los 4 años $(4,92 \pm 3,62)$ y entrenaban entre 2 y 3 horas/día, de 3 a 5 veces a las semana $(3,7 \pm 1,01)$.

Respecto a los criterios de inclusión, se utilizó un muestreo por conveniencia, centrándonos en gimnastas con edades comprendidas entre 12 y 18 años, debiendo cumplir con la firma de un consentimiento informado por parte de los padres, autorizando su participación voluntaria, excluyendo a quienes no firmaron el documento solicitado o no quisieron participar en las evaluaciones. En todo momento, se respetó las normas deontológicas expresadas en la

Declaración de Helsinki, siguiendo la normativa legal vigente española que regula la investigación clínica en humanos (Real Decreto 561/1993 sobre ensayos clínicos).

\section{Instrumentos de medidas}

\section{Medidas antropométricas}

La estatura fue medida utilizando un tallímetro (SECA220 con precisión de $1 \mathrm{~mm}$ ), con las gimnastas de pie y descalzadas, completamente estiradas, colocando los pies paralelos y 
con los talones unidos, las nalgas, hombros y cabeza en contacto con un plano vertical. La cabeza se mantuvo erguida con el borde orbitario inferior en el mismo plano horizontal que el conducto auditivo externo (plano de Frankfurt). El peso, se determinó con una balanza SECA (713, Hamburg, Alemania, con una precisión de $0.1 \mathrm{~kg}$ ). A raíz de los dos datos anteriores, se calculó el IMC como el peso dividido por la altura al cuadrado $(\mathrm{Kg} / \mathrm{m} 2)$. Al ser poblaciones adolescentes, se usó los valores indicados por Pan y Cole (Cole et al., 2007) delgadez grado III $(<16)$; delgadez grado II (16.1 a 17); delgadez grado I (17.1 a 18.5); normal (18.5 a 24.9), sobrepeso (25 a 30); y obesidad ( $\geq 30)$.

\section{Estima corporal}

La Estima Corporal fue determinada con el cuestionario Body-Esteem scale for children (BES-C) en su versión corta aplicados a niños y adolescentes (Méndelson y White 1982). La versión castellana del BES-C, indica una fiabilidad de .85 en Vernetta et al., (2011) y presenta una buena correlación con medidas de validez externa $(\mathrm{r}=0.68$ con el Piers-Harris Children's self-Concept Scale) (Piers et al., 2002). El cuestionario consta de 16 preguntas que valoran la estima corporal con posibilidad de respuestas dicotómicas SI/NO. El número de respuestas afirmativas y negativas para la alta estima es el mismo y cada una de ellas valen un punto. El valor de la escala se halla sumando el número de respuestas que indican alta estima.

\section{Procedimiento}

El estudio se llevó a cabo durante los meses de noviembre y diciembre del 2017. Primeramente se contactó con los entrenadores/as responsables de las gimnastas de diferentes clubs de Andalucía para solicitarles su participación y se les informó del objetivo del estudio Posteriormente, una vez obtenidos los permisos pertinentes y los consentimientos informados realizados por parte de los padres de las gimnastas, la mayoría menores de edad, se procedió a la toma de datos informándoles que su participación era voluntaria y las respuestas serían tratadas confidencialmente.

Las medidas fueron realizadas durante el entrenamiento en horario de tarde siguiendo siempre el mismo protocolo de actuación: las gimnastas rellenaban el cuestionario BES-C en una sala anexa mediante la presencia de sus entrenadores y dos autores de este trabajo para aclarar cualquier tipo de dudas y posteriormente, eran medidas en la misma sala con su ropa habitual de entrenamiento.

\section{Análisis estadístico}

Las variables cuantitativas se presentan con la media y la desviación típica y las categóricas por su frecuencia y porcentaje. La normalidad y homocedasticidad de las distribuciones se obtuvo a través de los estadístico Shapiro-Wilk y Levene respectivamente. Al no observar una distribución normal en parte de las distribuciones de los valores registrados de la Estima Corporal, según los distintos niveles de las variables IMC, Perímetro de cintura, y Edad se optó por un análisis no paramétrico. El contraste de muestras independientes, se ha realizado mediante la prueba $U$ de Mann Whitney. El nivel de significación fue de .05. Los datos se muestran en rangos promedio y todos fueron analizados utilizando SPSS, versión 22.0 (SPSS Inc., Chicago, IL, USA). 


\section{Resultados}

Tabla 1. Composición de la muestra y datos antropométricos.

\begin{tabular}{lccc}
\hline & $\begin{array}{c}\text { Acrobática } \\
(\mathrm{n}=34)\end{array}$ & $\begin{array}{c}\text { Rítmica } \\
(\mathrm{n}=33)\end{array}$ & $\begin{array}{c}\text { TOTAL } \\
(\mathrm{n}=67)\end{array}$ \\
\hline Edad (años) & $12.88( \pm 2.53)$ & $11.81( \pm 2.17)$ & $12.36( \pm 2.34)$ \\
Masa corporal $(\mathrm{Kg})$ & $42.68( \pm 10.91)$ & $37.40( \pm 8.60)$ & $40.08 \mathrm{Kg}( \pm 10.13)$ \\
Talla $(\mathrm{m})$ & $1.52( \pm .11)$ & $1.48( \pm .11)$ & $1.50 \mathrm{~m}( \pm .11)$ \\
IMC $\left(\mathrm{Kg} / \mathrm{m}^{2}\right)$ & $18.12( \pm 2.34)$ & $16.79( \pm 2.22)$ & $17.47( \pm 2.36)$ \\
PC $(\mathrm{cm})$ & $59.58( \pm 4.81)$ & $62.72( \pm 3.22)$ & $61.13( \pm 4.36)$ \\
\hline
\end{tabular}

En la Tabla 1 se muestran los datos descriptivos de las características de la muestra según la disciplina gimnásticas practicadas.

La Tabla 2 muestra el análisis descriptivo de cada uno de los ítems del BES-C, según la disciplina practicada y el total.

Tabla 2. Frecuencia (Porcentaje) de respuestas afirmativas a items del Cuestionario BES-C según disciplina practicada y total.

\begin{tabular}{lcccc}
\hline Ítem BES-C & G. Acrobática & G. Rítmica & Total & P (valor) \\
\hline Me gusta como salgo en las fotos & $34(100)$ & $24(72.7)$ & $58(86.6)$ & $.001^{*}$ \\
Estoy satisfecho con mi físico & $32(94.1)$ & $28(84.8)$ & $60(89.6)$ & .218 \\
No estoy contento con mi físico & $6(17.6)$ & $16(48.5)$ & $22(32.8)$ & $.008^{*}$ \\
Me gusto en el espejo & $34(100)$ & $32(97)$ & $66(98.5)$ & .310 \\
Ojalá estuviera más delgado & $2(5.9)$ & $4(12.1)$ & $6(9)$ & .375 \\
Cambiaría mi físico & $4(11.8)$ & $5(15.2)$ & $9(13.4)$ & .687 \\
Orgullosa de mi cuerpo & $34(100)$ & $31(93.9)$ & $65(97.0)$ & .148 \\
Satisfecho con mi peso & $33(97.1)$ & $25(75.8)$ & $58(86.6)$ & $.011^{*}$ \\
Me gustaría un físico mejor & $14(41.2)$ & $1(3.00)$ & $15(22.4)$ & $.000^{*}$ \\
Me avergüenzo de mi aspecto & $1(2.9)$ & 0 & $1(1.5)$ & .325 \\
Se burlan de mi aspecto & 0 & $3(9.1)$ & $3(4.5)$ & .074 \\
Creo que tengo buen cuerpo & $34(100)$ & $28(84.8)$ & $62(92.5)$ & $.019^{*}$ \\
Aspecto como me gusta & $34(100)$ & $29(87.9)$ & $63(94.0)$ & $.038^{*}$ \\
Buena opinión sobre mi aspecto & $34(100)$ & $29(87.9)$ & $63(94.0)$ & $.038^{*}$ \\
Aspecto tan bueno como los demás & $32(94.1)$ & $27(81.8)$ & $59(88.1)$ & .123 \\
A mis padres les gusta mi aspecto & $34(100)$ & $31(93.9)$ & $65(97.0)$ & .148 \\
\hline
\end{tabular}

(*) Nivel de significación .05

Respecto a la puntuación total del test. el estadístico U de Mann Whitney para muestras independientes mostró diferencias estadísticamente significativas entre los valores de rangos 
promedio de test BES-C y las gimnastas (rítmica y acrobática) $(\mathrm{U}=381.50 . \mathrm{p}=.018 . \mathrm{r}=-.29)$, (figura 1).

Figura 1. Rangos promedio de la puntuación total del test. Diferencias mostradas por la prueba U de Mann Whitney para muestras independientes.

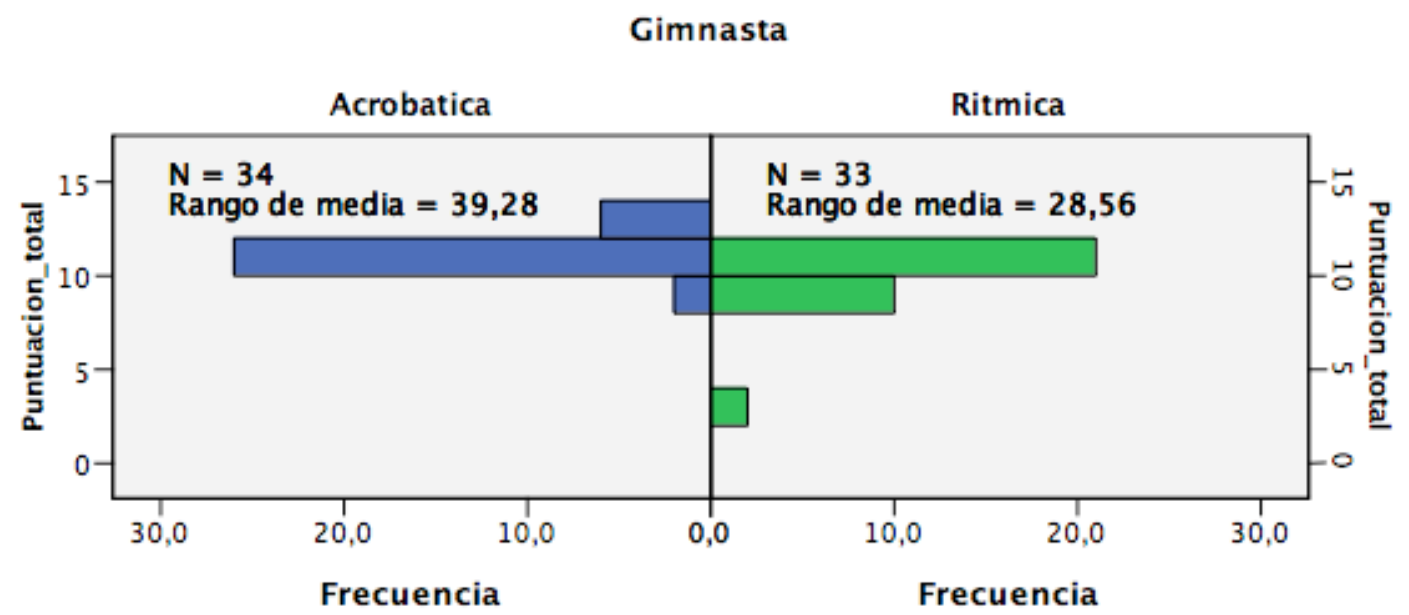

Igualmente se observan diferencias significativas entre las gimnastas de rítmica y acrobática con las variables IMC $(U=391.50, p=.035, r=-25.9)$ y perímetro de cintura $(U=809.00, p=$. $002, r=59.85)$, presentando las de acrobática, rangos promedios superiores en el IMC respecto a las de rítmica, siendo a la inversa en cuanto al perímetro de cintura (Figuras 2 y 3 ).

Figura 2. Rangos promedio del IMC. Diferencias mostradas por la prueba U de Mann Whitney para muestras independientes.

\section{Gimnasta}

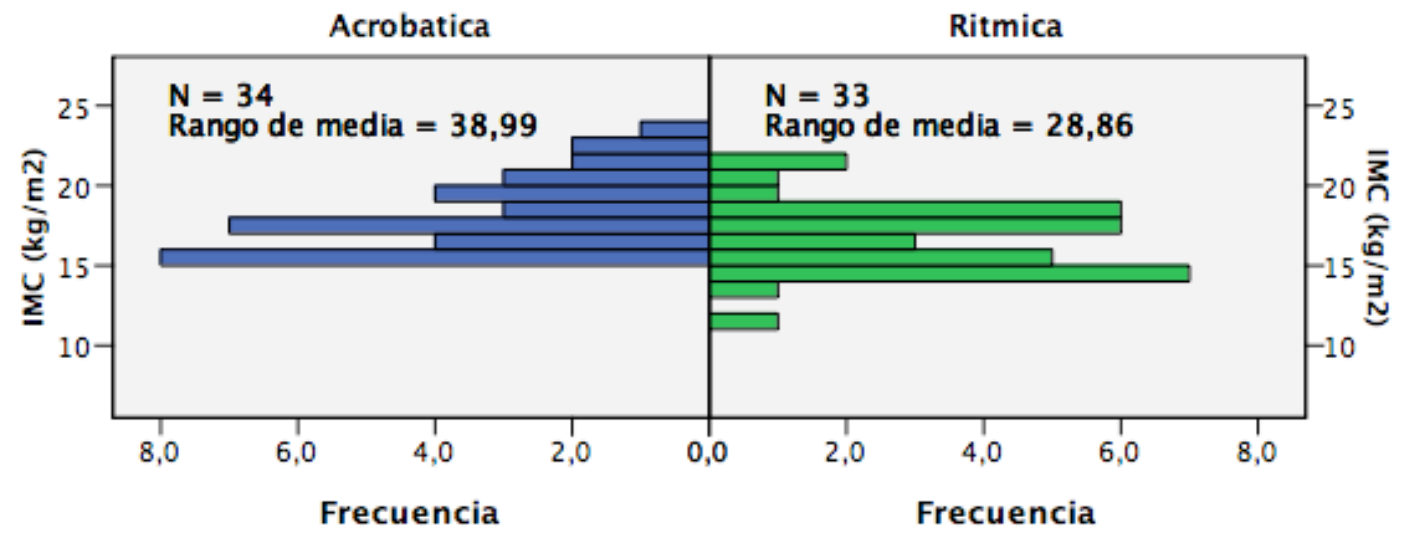


Figura 3. Rangos promedio del perímetro de cintura. Diferencias mostradas por la prueba U de Mann Whitney para muestras independientes.

\section{Gimnasta}

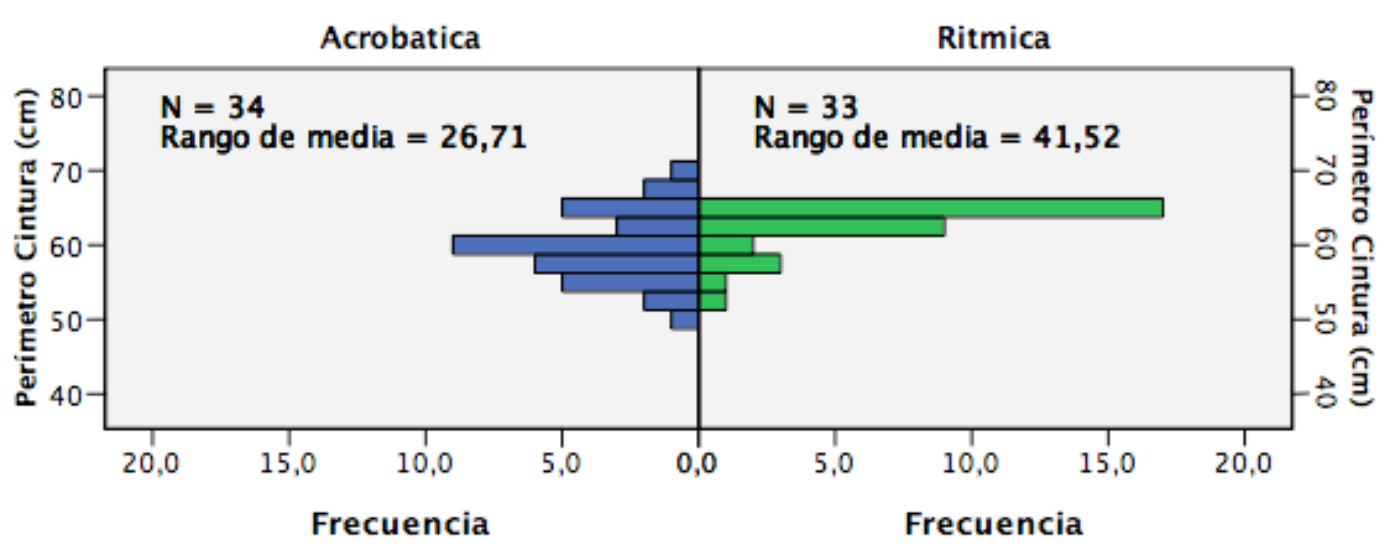

En la Tabla 3 se presenta el análisis de correlación, según disciplina gimnástica, realizado mediante el estadístico Rho de Spearman, entre las distintas variables. El estudio muestra como existe una correlación estadísticamente significativa $(p<.05$ y $\mathrm{p}<.01)$, entre IMC, perímetro de cintura, peso y talla.

Tabla 3. Análisis de correlación, según disciplina gimnástica. Estadístico Rho de Spearman

\begin{tabular}{|c|c|c|c|c|c|c|}
\hline & & Peso & Talla & IMC & $\begin{array}{l}\text { Perímetro } \\
\text { de cintura }\end{array}$ & $\begin{array}{c}\text { Test } \\
\text { BES-C }\end{array}$ \\
\hline \multirow{3}{*}{ Peso } & Rho de Spearman & & $.927^{* *}$ & $.892^{* *}$ & $.919^{* *}$ & .272 \\
\hline & Sig. (bilateral) & & .000 & .000 & .000 & .119 \\
\hline & $\mathrm{N}$ & & 34 & 34 & 34 & 34 \\
\hline \multirow{3}{*}{ Talla } & Rho de Spearman & $.847^{* *}$ & & $.696^{* *}$ & $.800^{* *}$ & .248 \\
\hline & Sig. (bilateral) & .000 & & .000 & .000 & .157 \\
\hline & $\mathrm{N}$ & 33 & & 34 & 34 & 34 \\
\hline \multirow{3}{*}{ IMC } & Rho de Spearman & $.768^{* *}$ & $.277^{*}$ & & $.902^{* *}$ & .311 \\
\hline & Sig. (bilateral) & .000 & .031 & & .000 & .073 \\
\hline & $\mathrm{N}$ & 33 & 33 & & 34 & 34 \\
\hline \multirow{3}{*}{$\begin{array}{l}\text { Perímetro } \\
\text { de cintura }\end{array}$} & Rho de Spearman & $.517^{* *}$ & $.412^{*}$ & $.447^{* *}$ & & .286 \\
\hline & Sig. (bilateral) & .002 & .017 & .009 & & .101 \\
\hline & $\mathrm{N}$ & 33 & 33 & 33 & & 34 \\
\hline \multirow{3}{*}{$\begin{array}{l}\text { Test } \\
\text { BES-C }\end{array}$} & Rho de Spearman & .087 & .002 & -.014 & -.046 & \\
\hline & Sig. (bilateral) & .629 & .991 & .937 & .799 & \\
\hline & $\mathrm{N}$ & 33 & 33 & 33 & 33 & \\
\hline
\end{tabular}

**. La correlación es significativa en el nivel .01 (2 colas).

*. La correlación es significativa en el nivel .05 (2 colas).
G. Acrobática
G. Rítmica 
El estudio de correlación considerando la muestra en su conjunto (Tabla 4), muestra como los diferentes test presenta una correlación de signo positivo entre todos los pares de las variables de peso, altura, perímetro de cintura e IMC.

Tabla 4. Análisis de correlación. Estadístico $R$ de Spearman de la muestra en su conjunto.

\begin{tabular}{ll|cccc}
\hline & Altura & IMC & $\begin{array}{c}\text { Perímetro } \\
\text { cintura }\end{array}$ & $\begin{array}{c}\text { Test BES- } \\
\text { C }\end{array}$ \\
\hline \multirow{3}{*}{ Peso } & Coeficiente de correlación & $.890^{* *}$ & $.885^{* *}$ & $.581^{* *}$ & .198 \\
& Sig. (bilateral) & .000 & .000 & .000 & .108 \\
& $\mathrm{~N}$ & 67 & 67 & 67 & 67 \\
\hline Altura & Coeficiente de correlación & & $.559^{* *}$ & $.551^{* *}$ & .154 \\
& Sig. (bilateral) & & .000 & .000 & .214 \\
& $\mathrm{~N}$ & & 67 & 67 & 67 \\
\hline \multirow{3}{*}{$\mathrm{IMC}$} & Coeficiente de correlación & & & $.513^{* *}$ & .185 \\
& Sig. (bilateral) & & & .000 & .133 \\
& $\mathrm{~N}$ & & & 67 & 67 \\
\hline
\end{tabular}

**La correlación es significativa en el nivel .01 (2 colas).

\section{Discusión}

El objetivo de este estudio de corte transversal fue ofrecer una aproximación a las diferencias y similitudes existente en relación a la estima corporal entre jóvenes practicantes de dos deportes de gran componente estético: gimnasia rítmica y gimnasia acrobática, así como su relación con el IMC y perímetro de cintura.

Los principales hallazgos, destacan en general, que las gimnastas incluidas en el estudio muestran buena estima corporal, existiendo una relación significativa con el IMC $(\mathrm{p}<.05)$.

Los resultados globales del BES-C mostraron una puntuación media ligeramente superior en GA (10.64 \pm 1.0$)$ que en GR $(9.48 \pm 1.95)$, pero sin diferencias significativas entre ambas disciplinas, destacando que la mayoría de las gimnastas se encuentran encuadradas en valores de buena estima corporal.

Teniendo en cuenta que las gimnastas de nuestro estudio, eran de la élite nacional, se corrobora la relación positiva existente entre los atletas que compiten en alto nivel, es decir, que tienen buen rendimiento competitivo y una mejor imagen corporal (Martínez- Gómez \& Veiga-Núñez, 2007; Kirkcaldy, Shephard, \& Siefen, 2002; Robinson \& Ferraro, 2004).

En línea con lo anterior, Hausenblas \& McNally (2004), indican que los deportistas suelen sentir una mayor satisfacción con su cuerpo que los que no deportistas (Hausenblas \& McNally, 2004).

Igualmente, nuestros datos son coincidentes con los estudios de gimnastas de acrobática y de rítmica donde los valores globales se encuentran entre 10.59 y 9.50 respectivamente (Pélaez \& Vernetta, 2018; Vernetta et al., 2011), alejándose de los trabajos que hallan alteración de la imagen corporal o menos satisfacción con su figura o imagen corporal en 
gimnastas y bailarinas, así como en atletas y jugadoras de hockey, fútbol y voleibol (Bass, Turner, \& Hunt, 2001; Meermann, 1997; Camacho et al., 2006; Valverde \& Moreno, 2016).

En el estudio de Camacho et al. (2016), se confirma que la imagen corporal depende fuertemente del tipo de deporte, estando la gimnasia rítmica entre los que mejores puntuaciones obtiene, datos que se constata en nuestra muestra.

El $100 \%$ de las gimnastas de acrobática respondieron positivamente a los ítems "Me gusta como salgo en las fotos", "Me gusto en el espejo", "Estoy orgullosa de mi cuerpo" y "Creo que tengo buen cuerpo", mientras que las de rítmica obtuvieron porcentajes ligeramente más bajos en los ítem anteriores $72.7 \%, 97.1 \%, 93.9 \%$ y $84.4 \%$, respectivamente. No existieron diferencias significativas en ninguna pregunta a excepción de "Me gusta como salgo en las fotos" y "Me gustaría tener un físico mejor" $(\mathrm{p}<.05)$ donde 9 de las 33 gimnastas de rítmica no le gusta como sale en las fotos y 14 de las 34 de acrobática le gustaría tener un físico mejor

Quizás esos porcentajes elevados, puede deberse a que las gimnastas de rítmica, aunque estén delgadas, tienen mayor preocupación por el perfeccionismo e insatisfacción general con su cuerpo como las bailarinas del estudio de Anshel (2004), de ahí, que cuando se ven en las fotos, tengan deseo de estar mejor.

De hecho, en nuestro trabajo, destacar que las gimnastas que practican rítmica muestran mayor preocupación en relación al peso, donde el $75.8 \%$ estaban satisfechas con su peso, frente al $97.1 \%$ de las de acrobática con diferencias significativas de .05. Estos resultados corroboran estudios previos que reportan como las gimnastas de rítmica, se perciben más sensibles a los cambios de peso pudiendo provocar una mayor disconformidad con la imagen corporal (Clavio \& Eagleman, 2011).

Igualmente, son coincidentes con el trabajo de Hernández-Alcántara et al. (2001), donde las gimnastas de rítmica con IMC bajo sobreestiman su imagen corporal mostrando un mayor apego a los cánones de delgadez.

No obstante, hay que resaltar, que aunque la preocupación fue mayor que las de acrobática, no existió diferencias entre ambas, siendo la Estima Corporal buena en ambas disciplinas.

Por otro lado, en cuanto a las gimnastas de acrobática, al ser aún la mayoría muy jóvenes y muchas de ellas pequeñas por el rol que desempeñan de ágiles, se encuentran aún desviadas de ese ideal respecto a su físico, de ahí, que el 41.17\%, es decir 14 de 34 gimnastas le gustaría mejorarlo.

En relación a los parámetros antropométricos, el IMC y el perímetro de cintura de la muestra en general fue de $17.47 \mathrm{~kg} / \mathrm{m}^{2}( \pm 2.36)$ y $61.13 \mathrm{~cm}( \pm 4.36)$ respectivamente, estando todas en delgadez grado I, según los valores de referencia (Cole et al., 2007). Las gimnastas de acrobática presentaron un IMC de $18.12( \pm 2.34)$ siendo similares a las gimnastas de esta disciplina del estudio de Peláez \& Vernetta (2018), con valores promedio de $18.06 \pm 2.19$ $\mathrm{kg} / \mathrm{m}^{2}$ y más altos que los $16.76 \mathrm{~kg} / \mathrm{m}^{2}$ y 16.13 de los de Taboada et al. (2017), en gimnastas de las modalidades de grupo y parejas femeninas respectivamente. Estos valores más bajos en estas gimnastas, pueden ser debido al propio proceso madurativo, ya que la edad en esas gimnastas era de $11.26( \pm 3.65)$ en modalidades de parejas y, 11.48 en grupos, siendo la edad de la muestra de nuestro estudio superior $(12.88 \pm 2.53)$. 
Igualmente, los valores presentados por las gimnastas de rítmica $16.79( \pm 2.22)$ son muy similares a los $16.17 \mathrm{~kg} / \mathrm{m}^{2}$ de Vernetta et al. (2011), pero más bajos que los reportados por Ávila-Carvalho, Klentrou, Palomero \& Lebre (2012) con $18.75 \mathrm{~kg} / \mathrm{m}^{2}$ y Montosa, Vernetta y López-Bedoya (2018) con $18.42( \pm 1.88)$.

Destacar que ninguna de nuestras gimnastas, muestran sobrepeso ni obesidad, datos que se alejan de los adolescentes españoles no deportistas de estas franja de edad donde el $4.2 \%$ en chicas escolares adolescentes entre 12 y 16 años padecían sobrepeso en el estudio de Doménech, Sánchez \& Ros (2015).

En cuanto al perímetro de cintura medio, en las gimnastas de acrobática fue de 59.58 $( \pm 4.81) \mathrm{cm}$ datos más bajos que los $62.15 \mathrm{~cm}$ que los de Peláez \& Vernetta (2008) en gimnastas de esta disciplina. Por otro lado, en las de rítmica fue $62.72( \pm 3.22) \mathrm{cm}$ similares a los $63.5 \mathrm{~cm}$ del estudios de Vernetta, Montosa y López-Bedoya (2018), y más elevado que los de Román, Del Campo, Solana \& Martín (2012) con $58.66 \mathrm{~cm}$. Todos estos datos, de nuestra gimnastas de ambas disciplinas, se alejan bastante de los $77.6 \mathrm{~cm}$ reportados en población normal de esta franja de edad (Arnaiz et al., 2010).

En general, los resultados más bajos del IMC y perímetro de cintura de estas gimnastas en relación a la población normal, guarda cierta relación con la importancia que estas deportistas otorgan al peso y su imagen corporal por ser un deporte estético donde la delgadez y la buena presencia constituyen factores importantes para poder ganar y tener éxito (Vernetta et al., 2011).

Igualmente destacar que la existencia de diferencias significativas entre ambas disciplinas en la variable IMC, muestra como los perfiles antropométricos en las gimnastas de rítmica correspondiente a una figura delgada, tiene su peso en este estudio, pudiendo esto por tanto ser reflejo en estas jóvenes deportistas.

En lo referente a la EC, los resultados de la muestra total mostraron una relación de signo positivo entre el IMC y la estima corporal $(p<.05)$. Estos mismos resultados ya se encontraron en gimnastas de rítmica y artística que afirman una relación inversa entre el IMC y EC, donde a menor peso, mayor estima corporal (Fernández, López \& López-Bedoya, 2001; Martínez, 1998; Vernetta et al., 2011).

Por último, nuestros resultados arrojaron la existencia de relaciones significativas de signo positivo, entre todos los pares de las variables antropométricas (peso, altura, perímetro de cintura e IMC Edad $(\mathrm{p}<.001)$. Estas relaciones ya se habían reportado anteriormente en adolescentes de gimnastas de acrobática debido al propio proceso madurativo (Peláez \& Vernetta, 2018).

Finalmente, este estudio presenta ciertas limitaciones que necesitan ser discutidas. La primera, el carácter transversal ya que no permite establecer relaciones de causa-efecto en las asociaciones entre EC y factores antropométricos, por lo que más estudios longitudinales se hacen necesarios para completar y verificar nuestros resultados. Por otro lado, en cuanto al IMC puede ser una variable poco útil según Kweitel (2007), en deportistas pues no aporta información sobre la masa magra o sobre la distribución de la grasa corporal, con lo cual, futuros estudios que calculen estos parámetros, podrían ser marcadores más adecuados para relacionarlos con el rendimiento. Igualmente, la representación muestral, que, al no ser muy grande en cada una de las disciplinas, abarcó franjas de edades muy amplias (de 12 a 18), siendo necesario en futuras investigaciones diferenciar por rangos de edades más cortos. 


\section{Conclusiones e implicaciones futuras}

Las gimnastas de ambas disciplinas presentan buena estima corporal con diferencias significativas entre ambas $(\mathrm{p}<.05)$, siendo las de acrobática las que mayor estima corporal poseen. Todas en general tienen buena opinión sobre su aspecto y su físico. El 12.1\% de las gimnastas de rítmica les gustaría estar más delgadas, a pesar de tener valores antropométricos bajos.

El valor medio del IMC de las gimnastas indicó normalidad nutricional o delgadez grado I, presentando las gimnastas de acrobática, rangos promedios superiores en el IMC respecto a las de rítmica. Igualmente, todas mostraron un perímetro de cintura por debajo de los valores referenciales existiendo diferencias significativas entre las gimnastas de acrobática y rítmica siendo éstas últimas las que presentan mayor perímetro de cintura. Se encontró relaciones significativas de signo positivo, entre todos los pares de las variables de peso, altura, perímetro de cintura e IMC $(\mathrm{p}<.001)$. Por último, la estima corporal de las de acrobática se asoció con un IMC saludable $(\mathrm{p}<.05)$.

En consecuencia, a los resultados, como aportaciones específicas, indicar que ambos deportes promueven una imagen corporal positiva. No obstante, es importante considerar que los porcentajes, aunque mínimos en algunos ítems de estas gimnastas que dan muestra de insatisfacción con su cuerpo o preocupación por su peso, obliga a los entrenadores a actuar en consecuencia, promoviendo medidas para el desarrollo de una imagen corporal positiva y saludable en estas gimnastas.

Futuras investigaciones deberían replicar estos hallazgos en muestras más amplias con otros rangos de edades, niveles diferentes y con otras disciplinas gimnásticas de componente artístico-estético. Igualmente, sería interesante el uso del Body Shape Questionnaire en su adaptación española de Raich, Mora, Marroquín, Pulido, \& Soler (1997), para analizar igualmente la EC en estas gimnastas y compararlo con el BES-C.

A raíz de estos resultados, sería conveniente continuar el proceso de investigación para generar propuestas de intervención específicas en estas gimnastas y a la postre, en otras disciplinas gimnásticas diferentes. Igualmente, sería interesante analizar la interacción de estas variables con otras relacionadas con la salud, como pueden ser la adherencia a la dieta mediterránea, las cantidades ingeridas el porcentaje graso, así como su relación con el rendimiento deportivo. Por último, es recomendable hacer estudios longitudinales y prospectivos, para constatar los resultados obtenidos en este trabajo.

\section{Referencias}

Aguilera, F. L. (2016). Estado nutricional, satisfacción con la imagen corporal e ingesta alimentaria e adolescentes que practican Gimnasia Artística. (Tesis de licenciatura). Universidad FASTA, Facultad de Ciencias Médicas.

Anshel, M.H. (2004): "Sources of Disordered Eating Patterns Between Ballet Dancers and Non-dancers". Journal of Sport Behaviour, 27(2), 115-133.

Arnaiz, P., Acevedo, M., Díaz, C., Bancalari, R., Barja, S., Aglony, M., Cavada, G., \& García, H. (2010). Razón cintura estatura como predictor de riesgo cardiometabólico en niños. Revista Chilena de Cardiología,, 29, 281-288. 
Ávila-Carvalho, L., Klentrou, P., Palomero, M.L., \& Lebre, E. (2012). Body composition profile of elite group rhythmic gymnasts. Science of Gymnastics Journal, 4(1), 21.

Bass, M., Turner, L., \& Hunt, S. (2001). Counseling of female athletes: Application of the stages of change model to avoid disordered eating, amenorrhea, and osteoporosis. Psychological Reports, 88, 1153-60.

Bobo-Arce, M., \& Méndez-Rial, B. (2013). Determinants of competitive performance in rhythmic gymnastics. a review. Journal of Human Sport and Exercise, 8(3), 711-727.

Camacho-Miñano, M. J., Fernández-García, E. \& Rodríguez-Galiano, M. I., (2006). Imagen corporal y práctica de actividad física en las chicas adolescentes: Incidencia de la modalidad deportiva. RICYDE: Revista Internacional de Ciencias del Deporte, 2(3), 1-19.

Campos, A., Pérez-Fabello, MaJ. \& Díaz, P. (2000). Gimnasia Ritmica: La imagen mental de novatos y expertos gimnastas. Revista de Psicología Del Deporte, 9(1-2), 87-93.

Clavio, G., \& Eagleman, A.N. (2011).Gender and sexually suggestive images in sports blogs. Journal of Sport Management, 7, 295-304.

Cole, T., Flegal, K., Nicholls, D. \& Jackson, A. (2007). Body mass index cut off sto define thinness in children and adolescents. International Survey, 335, 194-197.

Doménech, G., Sánchez, Á. \& Ros, G. (2015). Estudio transversal para evaluar los factores asociados a las diferencias entre la ciudad y los distritos de estudiantes de la escuela secundaria del sureste de España (Murcia) para su adhesión a la dieta mediterránea. Nutrición Hospitalaria, 31(3), 1359-1364.

Douda, H. T., Toubekis, A. G., Avloniti, A. A., \& Tokmakidis, S. P. (2008). Physiological and anthropometric determinants of rhythmic gymnastics performance. International Journal of Sports Physiology and Performance, 3(1), 41-54.

Esnaola, I. (2005). Autoconcepto físico y satisfacción corporal en mujeres adolescentes según el tipo de deporte practicado. Apunts Educación Física Y Deporte, 5-12.

Fernández, T. L. (2015). Relación entre el índice de masa corporal, la actividad física y los tiempos de comida en adolescentes mexicanos. Nutrición Hospitalaria, 32(3), 10821090. https://doi.org/10.3305/nh.2015.32.3.9331.

Hausenblas, H.A. y McNally, K.D. (2004): "Eating disoreder Prevalence and Symptoms for Track and Field Athletes and Nonathletes". Journal of Applied Sport Psychology, 16, 274-286.

Hernández-Alcántara, A., Aréchiga-Viramontes, J. \& Prado, C. (2009). Alteración de la imagen corporal en gimnastas. Archivos de Medicina Del Deporte, XXVI(130), 84-92.

Kirkcaldy, B. D., Shephard, R. J., \& Siefen, R. G. (2002). The relationship between physical activity and self-image and problem behaviour among adolescents. Social Psychiatry and Psychiatric Epidemiology, 37(11), 544-550.

Klentrou, P. \& Plyley, M. (2003). Onset of puberty, menstrual frequency, and body fat in elite rhythmic gymnasts compared with normal controls. Br. J. Sports Med, 37(6):490-4.

Kweitel, S. (2007). IMC: Herramienta poco útil para determinar el peso útil de un deportista. Revista Internacional de Medicina y Ciencias de la Actividad Física y Deporte 7(28), 274-289.

Martínez, C. (1998). Relaciones entre el clima motivacional, el bienestar psicológico y el rendimiento deportivo en la gimnasia rítmica y artística (Tesis Doctoral). Universidad de Valencia, Facultad de Psicología. 
Martínez-Gómez, D., \& Veiga-Núñez, O. L. (2007). Insatisfacción corporal en adolescentes: Relaciones con la actividad física e índice de masa corporal. Revista Internacional De Medicina Y Ciencias De La Actividad Física Y El Deporte, 7(27), 253-265.

Medelson, B., \& White, D. (1982). Relation between body-esteem and self-esteem of obese and normal children. Percept Mot Skills, 54(3), 899-905.

Meermann, R. (1997). Experimental investigation of dis- turbances in body image estimation in anorexia nervosa patients, and ballet and gymnastics pupils. International Journal of Eating Disorders, 2, 91-100.

Montosa, I., Vernetta, M. \& López-Bedoya, (2018). Assessment of health-related fitness by the ALPHA- fitness test battery in girls and adolescents who practise rhythmic gymnastics. Journal of Human Sport and Exercise, 13(1), 188-204.

Nordin S., Harris G. \& Cumming J. (2003). Disturbed eating in young, competitive gymnasts: Differences between three gymnastics disciplines. Eur J Sports Science, 3(5), 1-14.

Pélaez, E. \& Vernetta, M. (en prensa). Estima corporal y factores antropométricos en adolescentes practicantes de acrobática. Trances

Piers, E. V., Herzberg, D. S., \& Harris, D. B. (2002). Piers-Harris Children's Self-concept Scale:(PHCSCS). Western Psychological Services.

Purenović-Ivanović, T. \& Popović, R. (2014). Somatotype of Top-Level Serbian Rhythmic Gymnasts. J Hum Kinet, 03, 40, 181-187.

Raich, R. M., Mora, M., Marroquín, H., Pulido, A., \& Soler, A. (1997). Tratamiento cognitivoconductual de la insatisfacción corporal. Análisis y Modificaciones de Conducta, 23, 405-424.

Robinson, K., \& Ferraro, F. R. (2004). The relationship between types of female athletic participation and female body type. Journal of Psychology, 138(2), 115-128.

Román, M., Del Campo, V., Solana, R., \& Martín, J. (2012). Anthropometric and physical differences of the gymnasts from the talent identification program of the artistic and rhythmic specialtie. Retos: Nuevas Perspectivas de Educación Física, Deporte Y Recreación., 1(28), 58-62.

Salazar, Z. (2008). Adolescencia e imagen corporal en la época de la delgadez. Reflexiones. Universidad de Costa Rica, 87(2), 67-80.

Saucedo-Molina, T. J., Rodríguez, J., Oliva, L. A., Villarreal, M., León, R. C., \& Fernández, T. L. (2015). Relación entre el índice de masa corporal, la actividad física y los tiempos de comida en adolescentes mexicanos. Nutrición Hospitalaria, 32(3), 1082-1090. https://doi.org/10.3305/nh.2015.32.3.9331

Taboada-iglesias, Y., Vernetta, M. \& Gutiérrez-Sánchez, Á. (2017). Anthropometric Profile in Different Event Categories of Acrobatic Gymnastics. Journal of Human Kinetics, 57, 169-179. https://doi.org/10.1515/hukin-2017-005.

Vernetta, M., Fernández, E., López-Bedoya, J., Gómez-Landero, A., Oña, A. (2011). Estudio relacional entre el pérfil morfológico y estima corporal en la selección andaluza de gimnasia rítmica. Motricidad. European Journal of Human Movement, 26, 77-92.

Vernetta, M., López, J. \& Gutierrez, A. (2008). La creatividad en la Gimnasia Acrobática. En Martínez, A. \& Díaz, P (Coord). Creatividad y Deporte: consideraciones teóricas e investigaciones breves. Sevilla, Wanceulen, pp.133-154. 
Vernetta, M., Montosa, I., \& López-Bedoya, J. (2018). Dieta Mediterránea en jóvenes practicantes de gimnasia rítmica. Revista Chilena de Nutrición, 45(1), 37-44.

Vernetta, M., Montosa, I., Jiménez-Beas, J.D., \& López-Bedoya, J. (2017) Batería Funcional ARISTO en Gimnasia Rítmica: protocolo de test específicos para la evaluación de jóvenes gimnastas en un ámbito de entrenamiento saludable. Revista Andaluza de Medicina del Deporte, 10(3), 112-119.

Valverde, P. \& Moreno, S. (2016). Percepción de la imagen corporal en mujeres jóvenes deportistas (Trabajo fin de grado). Universidad de Jaén, Facultad de Humanidades y Ciencias de la Educación. 